\title{
TABLE OF CASES AND OPINIONS
}

\section{EUROPEAN COURTS}

\section{Court of Justice of the European Union}

\section{Court of Justice}

Abdoulaye Amadou Tall v Centre public d'action sociale de Huy (case C-239/14)

EU:C:2015:824

Acciaieria Ferriera di Roma (FERAM) and others v High Authority of the ECSC (joined cases 9 and 25/64) 91965] ECR 311

Administration des Douanes et Droits Indirects v Legros and Others (case C-163/90) [1992] ECR I-4625

AGM-COS MET Srl v Suomen valtio and Tarmo Lehtinen (case C-470/03) [2007] ECR I-2749 $6.054,6.071$

Agrana Zucker GmbH v Bundesminister für Land-und Forstwirtschaft, Umwelt und

Wasserwirtschaft (case C-309/10) [2011] ECR I-7333 4.023

Agraz, SA and Others v Commission of the European Communities (case C-243/05 P) [2006] ECR I-10833

Åklagaren v Hans Åkerberg Fransson (case C-617/10) EU:C:2013:105 $2.023,2.031$

Aktien-Zuckerfabrik Schöppenstedt v Council of the European Communities (case 5/71) [1971] ECR 975 $3.012,3.013,3.017,3.018,3.026,4.026$

Akzo Nobel Chemicals Ltd and Akcros Chemicals Ltd v European Commission (case

C-550/07 P) [2010] ECR I-8301 2.055

Alakor Gabonatermelö és Forgalmazó Kft v Nemzeti Adó- és Vámhivatal Észak-alföldi Regionális Adó Föigazgatósága (case C-191/12) EU:C:2013:315 .... 2.060, 5.012, 5.042, $5.129-5.134,5.173,5.174,5.178$

Alfons Lütticke GmbH v Commission of the European Communities (case 4/69) [1971] ECR 325 $3.009,3.011$

Alfredo Grifoni v European Atomic Energy Community (case C-308/87) [1990] ECR I-1203 $3.020,3.075$

Alo Heinemann v Commission of the European Communities (case 79/71) [1972] ECR 579 3.078

Amministrazione delle Finanze dello Stato v Denkavit Italiana Srl (case 61/79) [1980] ECR 1205 5.083-5.088, 9.023

Amministrazione delle Finanze dello Stato v Simmenthal SpA (case 106/77) [1978] ECR 629 7.023

Amministrazione delle Finanze dello Stato v SpA San Giorgio (case 199/82) [1983] ECR 3595 2.068, 3.107, 4.029, 5.012, 5.014, 5.029, 5.064, 5.066, 5.083, 5.093-5.100, $5.101,5.103,5.108,5.140,5.141,5.149,5.150,5.155,5.158,5.161,7.216,8.046,9.045$ Anne Muller (née Collignon) v Commission of the European Communities (case 4/67) [1967] ECR 469 3.031 
Ansaldo Energia SpA v Amministrazione delle Finanze dello Stato, Amministrazione delle Finanze dello Stato v Marine Insurance Consultants Srl and GMB Srl and Others v Amministrazione delle Finanze dello Stato (joined cases C-279/96-C-281/96) [1998] ECR I-5025 $2.028,2.61,2.062,2.063$

Antonio Muñoz y Cia SA and Superior Fruiticola SA v Frumar Ltd and Redbridge Produce Marketing Ltd (case C-253/00) [2002] ECR I-7289 $8.009,8.011$ Apple and Pear Development Council v KJ Lewis Ltd and others (case 222/82) [1983] ECR 4083 5.126, 5.167, 5.171, 5.172, 5.174

Artegodan GmbH v European Commission and Federal Republic of Germany (case C-221/10 P) EU:C:2012:216 3.018

Asociación Nacional de Establecimientos Financieros de Crédito (ASNEF) and Federación de Comercio Electrónico y Marketing Directo (FECEMD) v Administración del Estado (joined cases C-468/10 and C-469/10) [2011] ECR I-12181 $2.045,2.046$

Association de médiation sociale v Union locale des syndicats CGT and Others (case C-176/12) EU:C:2014:2

Asociația Accept v CNCD (case C-81/12) EU:C:2013:275 $2.023,2.024$

Asteris $\mathrm{AE}$ and others v Hellenic Republic and European Economic Community (joined cases 106/87-120/87) [1988] ECR 5515 3.008

Banca Antoniana Popolare Veneta SpA v Ministero dell'Economia e delle Finanze and Agenzia delle Entrate (case C-427/10) [2011] ECR I-13377 5.024-5.026, 7.062

Bayerische HNL Vermehrungsbetriebe GmbH \& Co KG and others v Council and Commission of the European Communities (joined cases 83 and 94/76, 4, 15 and 40/77) [1978] ECR 1209 $3.012,6.017$

Bela-Mühle Josef Bergmann KG v Grows-Farm GmbH \& CO KG (case 114/76) [1977] ECR 1211 5.089

Belgische Radio en Televisie (BRT) and société belge des auteurs, compositeurs et éditeurs v SV SABAM and NV Fonior (case 127/73) [1974] ECR 51 7.011-7.013, 8.009, 8.011

Bent Jensen and Korn-og Foderstofkompagniet A/S v Landbrugsministeriet EF-Direktoratet (case C-132/95) [1998] ECR I-2975 $5.167,5.172$

Birra Wührer SpA and others v Council and Commission of the European Communities (joined cases 256, 257, 265, 267/80, 5 and 51/81 and 282/82) [1984] ECR 3693 3.073, 3.074, 3.092, 3.095, 3.096, 3.097, 3.098, 3.099, 3.101, 3.109, 4.033, $9.014,9.23$

BNO Walrave and LJN Koch v Association Union cycliste internationale, Koninklijke Nederlandsche Wielren Unie and Federación Española Ciclismo (case 36/74) [1974] ECR 1405 $8.011,8.037$

Booker Aquacultur Ltd and Hydro Seafood GSP Ltd v The Scottish Ministers (joined cases C-20/00 and C-64/00) [2003] ECR I-7411 2.043

Braathens Sverige AB v Riksskatteverket (case C-346/97) [1999] ECR I-3419 ............. 5.030

Brasserie du Pêcheur SA v Bundesrepublik Deutschland and The Queen v Secretary of State for Transport, ex parte: Factortame Ltd and others (joined cases C-46/93 and C-48/93) [1996] ECR I-1029 .......... 2.058, 3.010, 3.013, 3.039, 3.071, 5.007, 5.115, 6.009, 6.010, 6.012, 6.013, 6.017, 6.018, 6.020, 6.021, 6.024, 6.028, 6.029, 6.053, 6.053, 6.054, 6.070, 6.072, 6.082-6.084, 6.087, 6.089, 8.016, 9.045

Brinkmann Tabakfabriken GmbH v Skatteministeriet (case C-319/96) [1998] ECR $\mathrm{I}-5255$ $6.054-6.058,6.063,6.071$ 
Bruno Barra v Belgian State and City of Liège (case 309/85) [1988] ECR 355 ............. 8.037

BS Levez v T H Jennings (Harlow Pools) Ltd (case C-326/96) [1998] ECR I-7835 ... 2.064, 2.066, 5.019, 5.020, 5.027, 6.039, 7.057, 8.025

Carmine Antonio Russo v Azienda di Stato per gli interventi sul mercato agricolo (AIMA) (case 60/75) [1976] ECR 45 6.004

City Motors Groep NV v Citroën Belux NV (case C-421/05) [2007] ECR I-653 ....... 7.134,

CMC Cooperativa muratori e cementisti and others v Commission of the European

Communities (case 118/83) [1985] ECR 2325 ........... 3.034, 3.036, 3.062, 6.078, 7.125,

Combinatie Spijker Infrabouw-De Jonge Konstruktie and Others v Provincie Drenthe (case

C-568/08) [2010] ECR I-12655 $6.081,6.088$

Comet BV v Produktschap voor Siergewassen (case 45/76) [1976] ECR 2043 ... 2.008, 2.053, $2.054,2.061,5.008,5.014,5.078,5.081$

Commission of the European Communities v AssiDomän Kraft Products AB, Iggesunds

Bruk AB, Korsnäs AB, MoDo Paper AB, Södra Cell AB, Stora Kopparbergs Bergslags

AB and Svenska Cellulosa AB (case C-310/97 P) [1999] ECR I-5363 4.012

Commission of the European Communities v Camar Srl and Tico Srl (case C-312/00 P)

[2002] ECR I-11355 3.016

Commission of the European Communities v CEVA Santé Animale SA and Pfizer

Enterprises Sàrl (case C-198/03 P) [2005] ECR I-6357

Commission of the European Communities v Federal Republic of Germany (case C-102/96)

[1998] ECR I-6871 6.030

Commission of the European Communities v French Republic (case C-232/05) [2006] ECR I-10071 2.055

Commission of the European Communities v Fresh Marine Company A/S (case C-472/00

P) [2003] ECR I-7541 3.059

Commission of the European Communities v Italian Republic (case 104/860 [1988] ECR 1799

Commission of the European Communities v Hellenic Republic (case 68/88) [1989] ECR

2965

Commission of the European Communities v Italian Republic (case C-437/01) [2003] ECR

I-9861 5.030

Commission of the European Communities v Italian Republic (case C-129/00) [2003] ECR I-14637 $5.142,5.149$

Commission of the European Communities v Schneider Electric SA (case C-440/07 P)

[2009] ECR I-6413 $3.048,7.137$

Compagnie Continentale France v Council of the European Communities (case 169/73)

[1975] ECR 117

$3.053,3.068$

Comptoir national technique agricole (CNTA) SA v Commission of the European

Communities (case 74/74) [1976] ECR 797

Costa v ENEL (case 6/640) [1964] ECR 585

Cotter, Ann and Norah McDermott v Minister for Social Welfare and Attorney General

(case C-377/89) [1991] ECR I-1155 $5.167-5.180$ 
Courage Ltd v Bernard Crehan and Bernard Crehan v Courage Ltd and Others (case C-453/99) [2001] ECR I-6297 ............ 1.012, 2.004, 2.019, 2.027, 2.060, 5.164, 7.004, 7.011, 7.015-7.022, 7.023, 7.024, 7.039, 7.052, 7.054, 7.061, 7.065, 7.105, 7.119, 7.125, 7.130, 7.170-7.172, 7.173, 7.174-7.177, 7.178, 7.181, 7.225, 7.243, 8.010, 8.032, 8.036, 9.014, 9.057

Danfoss A/S and Sauer-Danfoss ApS v Skatteministeriet (Case C-94/10) [2011] ECR I-9963 ......... 1.012, 1.016, 1.032, 2.060, 3.032, 3.037, 3.039, 3.068, 4.027, 4.030, 5.007, 5.012, 5.015, 5.016, 5.027, 5.029, 5.030-5.044, 5.048, 5.049, 5.050, 5.052, 5.054, 5.057, $5.059,5.060,5.061,5.131,5.179,5.180,5.182,5.183,6.002,6.003,6.025,6.041-6.051$, 6.054, 6.072, 6.075-6.078, 6.091, 6.094, 6.095, 7.145, 8.026, 8.043, 8.046, 9.004, 9.005, $9.007,9.011,9.012,9.019,9.022,9.024,9.033,9.036,9.067$

Dansk Denkavit ApS and P Poulsen Trading ApS, supported by Monsanto-Searle A/S v Skatteministeriet (case C-200/90) [1992] ECR I-2217 ......... 5.124, 5.136, 5.155, 5.161

Dansk Industri (DI), acting on behalf of Ajos A/S v Estate of Karsten Eigil Rasmussen (case C-441/14) EU:C:2016:278 $8.009,8.038,8.039$

Danske Slagterier v Bundesrepublik Deutschland (case C-445/06) [2009] ECR I-2119 $6.026,6.029,6.030,6.031,6.034,6.038,6.040,6.070,6.086$

DEB Deutsche Energiehandels- und Beratungsgesellschaft mbH v Bundesrepublik Deutschland (case 279/09) [2010] ECR I-13849 2.006, 2.008, 2.037

Développement SA and Clemessy v Commission of the European Communities (case 267/82) [1986] ECR 1907 3.034

DGV, Deutsche Getreideverwertung und Rheinische Kraftfutterwerke $\mathrm{GmbH}$ and others v Council and Commission of the European Communities (joined cases 241, 242, 245 to 250/78) [1979] ECR 3017 $1.025,1.032,3.047,3.084,3.090,3.091,3.103,9.014$ $9.021,9.023$

Digital Rights Ireland Ltd (case C-293/12) v Minister for Communications, Marine and Natural Resources and Others and Kärntner Landesregierung (C-594/12) and Others EU:C:2014:238 2.048, 2.049

Dilexport Srl v Amministrazione delle Finanzo dello Stato (case C-343/96) [1999] ECR I-579 $5.019,5.020,5.116,5.141,5.143$

Draehmpaehl, Nils v Urania Immobilienservice OHG (case C-180/95) [1997] ECR I-2195 $2.023,2.024,2.025,6.085$

Dragoş Constantin Târşia v Statul român, Serviciul public comunitar regim permise de conducere şi înmatriculare a autovehiculelor (case C-69/14) EU:C:2015:662 ........ 2.062,

5.014

Edilizia Industriale Siderurgica Srl (Edis) v Ministero delle Finanze (case C-231/96) [1998] ECR I-4951 $2.028,2.061,2.062,2.063,5.014,5.019,7.059$

E.ON Földgáz Trade Zrt v Magyar Energetikai és Közmü-szabályozási Hivatal (case C-510/13) EU:C:2015:189 $2.002,2.036$

ÉR, OO, JR and Others v Council of the European Union and Commission of the European Communities (case C-100/07 P) [2007] ECR I-136 3.009

Erich Dillenkofer, Christian Erdmann, Hans-Jürgen Schulte, Anke Heuer, Werner, Ursula and Torsten Knor v Bundesrepublik Deutschland (joined cases C-178/94, C-179/94, C-188/94, C-189/94 and C-190/94) [1996] ECR I-4845 .... 3.013, 3.015, 6.013-6.016, 6.017

European Commission v Federal Republic of Germany (case C-271/08) [2010] ECR I-7091 2.046 
European Commission v United Kingdom of Great Britain and Northern Ireland (case

C-640/13) EU:C:2014:2457 5.026

European Parliament v Council of the European Union (case C-355/10)

EU:C:2012:516 2.041

Europese Gemeenschap v Otis NV and Others (case C-199/11) EU:C:2012:684 ........ 2.033, 7.034, 7.054, 7.056, 7.105, 7.134, 7.135, 7.136, 8.011

Evangelischer Krankenhausverein Wien v Abgabenberufungskommission Wien and Wein \&

Co HandelsgesmbH v Oberösterreichische Landesregierung (case C-437/97) [2000]

ECR I-1157

Express Dairy Foods Ltd v Intervention Board for Agricultural Produce (case 130/79) [1980] ECR 1887 5.012, 5.146-5.148

Fantask A/S ea v Industriministeriet (Erhvervministeriet) (case C-188/95) [1997] ECR I-6783 $2.062,5.014,5.024,5.172,6.029$

FIAMM SpA and FIAMM Technologies LLC, Giorgio Fedon and Figli SpA and Fedon

America, Inc. v Council of the European Union and Commission of the European

Communities (joined cases C-120/06 P and C-121/06 P) [2008] ECR I-6513 ..... 3.021

Filomena Califano v Wind SpA (case C-318/08) [2010] ECR I-2213 2.006

Finanziaria Siderurgica Finsider SpA (in liquidation), Italsider SpA (in liquidation) and Societa Acciaierie e Ferriere Lombarde Falck SpA v Commission of the European Communities (joined cases C-363/88 and C-364/88) [1992] ECR I-359 3.043

Firma E Kampffmeyer and others v Commission of the EEC (joined cases 5, 7 and 13 to 24/66) [1967] ECR 245, 260 and 262 3.057, 3.078, 3.080, 4.008

Foster, A and others v British Gas plc (case C-188/89) [1990] ECR I-3313 ...... 1.047, 6.020, $7.001,8.001,9.007$

Françoise-Eléonor Hanssens-Ensch v European Community (case C-377/09) [2010] ECR I-7751

Francovich and Bonifaci and others v Italian Republic (joined cases C-6/90 and 9/90) [1991] ECR I-5357 ............ 2.004, 2019, 2.027, 2.060, 3.010, 3.013, 5.007, 5.016, 6.004, 6.005, 6.006, 6.007, 6.008, 6.009, 6.010-6.012, 6.013, 6.026, 6.037, 6.054, 7.013, 7.023, 9.045

GAARM v Commission of the European Communities (case 289/83) [1984] ECR 4295 $1.012,3.025$

Gabrielle Defrenne v Société anonyme belge de navigation aérienne Sabena (Defrenne II) (case 43/75) [1976] ECR 455 $8.009,8.011$

Gaetano Donà v Mario Mantero (case 13/76) [1976] ECR 1333 8.011

Gerhard Köbler v Republik Österreich (case C-224/01) [2003] ECR I-10239 .... 3.016, 6.022

Gerhardus Leussink and others v Commission of the European Communities (joined cases 169/83 and 136/84) [1986] ECR 2801 3.062

Grand Duchy of Luxemburg v Berthe Linster, Aloyse Linster and Yvonne Linster (case C-287/98) [2000] ECR I-6917 8.038

Graphic Procédé v Ministère du Budget, des Comptes publics et de la Fonction publique (case C-88/09) [2010] ECR I-1049 5.043

GT-Link A/S v De Danske Statsbaner (DSB) (case C-242/95) [1997] ECR I-4449 ... 1.047, $5.014,8.036$

Guérin Automobiles v Commission of the European Communities (case C-282/95 P) [1997] ECR I-1503 $7.013,7.014$

Günter Fuß v Stadt Halle (case C-429/09) [2010] ECR I-12167 ............ 6.018, 6.032, 6.033, $6.035,6.054,6.070,6.071,6.081$ 
Haahr Petroleum Ltd v Åbenrå Havn, Ålborg Havn, Horsens Havn, Kastrup Havn NKE A/S, Næstved Havn, Odense Havn, Struer Havn and Vejle Havn, and Trafikministeriet (case C-90/94) [1997] ECR I-4085 $2.062,5.014,5.024,5.172$

Hans Just I/S v Danish Ministry for Fiscal Affairs (case 68/79) [1980] ECR 501 ........ 5.011, 5.012, 5.013, 5.076-5.083, 5.085, 5.086, 5.088, 5.097, 5.136, 5.142, 5.144, 5.150, 5.155, $5.157,5.158,5.161,5.162,7.173,9.021,9.045,9.059$

Hartmut Eifert v Land Hessen (case C-93/09) [2010] ECR I-11063 ..... 2.041

Hellenic Republic v Commission of the European Communities (case C-259/87) [1990] ECR I-2845 $4.016,4.018$

Herbert Schaible v Land Baden-Württemberg (case C-101/120) EU:C:2013:661 ......... 2.050 H J Banks \& Co Ltd v British Coal Corporation (case C-128/92) [1994] ECR I-1209 $7.013,7.52,8.017$

Holcim (Romania) SA v European Commission (case C-556/14 P) EU:C:2016:207 .... 3.022 Ikea Wholesale Ltd v Commissioners of Customs and Excise (case C-351/04) [2007] ECR I-7723 4.007

Ilie Nicolae Nicula v Administrația Finanțelor Publice a Municipiului Sibiu and Administraţia Fondului pentru Mediu (case C-331/13) EU:C:2014:2285 5.128 $5.134,5.175-5.178$

Impact v Minister for Agriculture and Food and Others (case C-268/06) [2008] ECR I-2483 2.006

Industrie- en Handelsonderneming Vreugdenhil BV v Commission of the European Communities (case C-282/90) [1992] ECR I-1937 4.007

International Transport Workers' Federation and Finnish Seamen's Union v Viking Line ABP and OÜ Viking Line Eesti (case C-438/050) [2007] ECR I-10779 ... 8.011

Interquell Stärke-Chemie GmbH and Diamalt AG v Council and Commission of the European Communities (joined cases 261 and 262/78) [1979] ECR 3045 ........... 1.025, 1.032, 3.074, 3.078, 3.084, 3.085, 3.091, 3.099, 3.103, 3.111, 9.014, 9.021, 9.023, 9.059

Inuit Tapiriit Kanatami and Others v European Parliament and Council of the European Union (case C-583/11 P) EU:C:2013:625 $2.019,2.020,5.047,6.049,8.013$

Ioan Tatu v Statul român prin Ministerul Finanțelor ss i Economiei and Others (case C-402/09) [2011] ECR I-2711 5.176

Ireks-Arkady GmbH v Council and Commission of the European Communities (case 238/78) [1979] ECR 2955 .......... 1.025, 1.032, 3.025, 3.072, 3.073, 3.074, 3.084, 3.086, $3.087,3.088,3.089$, 3.090, 3.099, 3.100, 3.102, 3.103, 3.109, 3.113, 7.173, 9.014, 9.021, $9.023,9.026,9.030,9.059$

J C J Wouters, J W Savelbergh and PriceWaterhouse Belastingadviseurs BV v Algemene Raad van de Nederlandse Orde van Advocaten, intervener: Raad van de Balies van de Europese Gemeenschap (case C-309/99) [2002] ECR I-1577 8.011

Jean-E Humblet v Belgian State (case 6/60) [1960] ECR 559 $5.008,6.004$

Jeroen van Schijndel and Johannes Nicolaas Cornelis van Veen v Stichting Pensioenfonds voor Fysiotherapeuten (joined cases C-430/93 and C-431/930 [1995] ECR I-4705 $2.069,2.070,2.073,5.022,7.060,8.026$

J M Mulder, W H Brinkhoff, J M M Muskens, T Twijnstra and Otto Heinemann v Council of the European Union and Commission of the European Communities (joined cases C-104/89 and C-37/90) [2000] ECR I-203 $3.071,3.073,3.081$ 
J M Mulder W H Brinkhoff, J M M Muskens, T Twijnstra and Otto Heinemann v Council of the European Communities and Commission of the European Communities (joined cases C-104/89 and C-37/90) [1992] ECR I-3061 $3.081,3.103$

J N v Staatssecretaris van Veiligheid en Justitie (case C-601/15 PPU) EU:C:2016:84 ... 2.041, $2.043,2.044,2.045,2.046$

Johnston, Marguerite v Chief Constable of the Royal Ulster Constabulary (case 222/84) [1986] ECR 1651 $2.008,2.030,2.034$

Jutta Leth v Republik Österreich and Land Niederösterreich (case C-420/11) EU:C:2013:166 6.054, 6.064-6.069, 6.071, 7.134

Kapniki Michailidis AE v Idryma Koinonikon Asfaliseon (IKA) (joined cases C-441/98 and C-442/98) [2000] ECR I-7145 ... 5.012, 5.116, 5.117, 5.137, 5.143, 5.184, 5.185, 7.173

Kiriaki Angelidaki and Others v Organismos Nomarchiakis Autodioikisis Rethymnis and Others (joined cases C-378/07-C-380/07) [2009] ECR I-3071 2.055

Knauf Gips KG v European Commission (case C-407/08 P) [2010] ECR I-6375 ........ 2.041

Kone AG and Others v ÖBB-Infrastruktur AG (case C-557/12) EU:C:2014:1317 ...... 1.012, 1.016, $6.068,7.034-7.038,7.050,7.105,7.119,7.121,7.124,7.132,7.136$, $7.137-7.145,7.146,7.148,7.149,8.028,8.029,9.010$

Kurt Kampffmeyer Mühlenvereinigung KG and others v Commission and Council of the

European Communities (joined cases 56-60/74) [1976] ECR 711 $3.074,3.076$

Laboratoires pharmaceutiques Bergaderm SA and Jean-Jacques Goupil v Commission of the

European Communities (case C-352/98 P) [2000] ECR I-5291 ..... 3.010, 3.011, 3.014, 3.015, 3.017, 3.018, 3.012, 3.043, 3.057, 6.072, 7.134

Lady \& Kid A/S and Others v Skatteministeriet (case C-398/09) [2011] ECR

I-7375 5.023, 5.064, 5.123-5.129, 5.134, 5.171, 5.172, 5.173, 5.174, 5.178

Laval un Partneri Ltd v Svenska Byggnadsarbetareförbundet, Svenska

Byggnadsarbetareförbundets avdelning 1, Byggettan and Svenska Elektrikerförbundet (case C-341/05) [2007] ECR I-11767 $8.002,8.017,8.037$

Léger, Geoffrey v Ministre des Affaires sociales, de la Santé et des Droits des femmes and

Etablissement français du sang (case C-528/13) EU:C:2015:288 ..... 2.042, 2.043, 2.044,

$2.045,2.046$

Liivimaa Lihaveis MTÜ v Eesti-Läti programmi 2007-2013 Seirekomitee (case C-562/12)

EU:C:2014:2229 2.042

Liselotte Hauer v Land Rheinland-Pfalz (case 44/79) [1979] ECR 3727 2.043

Littlewoods Retail Ltd and Others v Her Majesty's Commissioners of Revenue and Customs (case C-591/10) EU:C:2012:478 $5.068,5.071$

Manfredi, Vincenzo v Lloyd Adriatico Assicurazioni SpA, Antonio Cannito v Fondiaria Sai $\mathrm{SpA}$ and Nicolò Tricarico and Pasqualina Murgolo v Assitalia SpA (joined cases C-295/04-C-298/04) [2006] ECR I-6619. $2.058,5.164,6.038,6.053,7.025$ 7.026-7.033, 7.034, 7.040, 7.052, 7.054, 7.061, 7.062, 7.105, 7.119, 7.125, 7.130-7.132, $7.133,7.134,7.145,7.156,7.157,7.159,7.165,7.178-7.180,7.181,7.225,8.028,8.030$, $8.032,8.043,9.014,9.057$

Mannesmann AG and others v High Authority of the European Coal and Steel Community (joined cases 4/59-13/59) [1960] ECR 113 4.003

Mariana Irimie v Administrația Finanțelor Publice Sibiu et Administraţia Fondului pentru Mediu (case C-565/11) EU:C:2013:250 $5.068,5.071$ 
Marshall, M Helen v Southampton and South-West Hampshire Area Health Authority (Marshall II) (case C-271/91) [1993] ECR I-4367 .... 2.023, 2.024, 6.083, 6.085, 6.087, $6.088,7.119$

Masdar (UK) Ltd v Commission of the European Communities (case C-47/07 P) [2008] ECR I-9761 ........... 2.004, 2.016, 4.002, 4.004, 4.017, 4.018, 4.019-4.021, 4.022, 4.023, $4.024,4.028,4.035,5.086,9.045$

Metallgesellschaft Ltd and Others, Hoechst AG and Hoechst (UK) Ltd v Commissioners of Inland Revenue and HM Attorney General (joined cases C-397/98 and C-410/98) [2001] ECR I-1727 .......... 2.036, 5.007, 5.045, 5.069, 5.070, 5.071, 6.031, 6.035, 6.086, 6.088

Ministre du Budget, des Comptes publics et de la Fonction publique v Accor SA (case C-310/09) [2011] ECR I-8115 $5.007,5.012,5.129,5.134,5.172,5.178$

Multiservice Srl v Telecom Italia SpA (case C-320/08) [2010] ECR I-2213 ...... 2.006

Neptune Distribution SNC v Ministre de l'Économie et des Finances (case C-157/14) EU:C:2015:823 $2.041,2.043,2.046,2.048$

NV Algemene Transport- en Expeditie Onderneming van Gend \& Loos v Netherlands Inland Revenue Administration (case 26/62) [1963] ECR 1 2.053, 7.001, 7.002,

Paola Faccini Dori v Recreb Srl (case C-91/92) [1994] ECR I-3325 6.008

Paquay, Nadine v Société d'architects Hoet + Minne SPRL (case C-460/06) [2007] ECR I-8511 2.024

PARAT Automotive Cabrio Textiltetőket Gyártó Kft v Adó- és P.nzügyi Ellenőrzési Hivatal, Hatósági Főosztály, Észak-magyarországi Kihelyezett Hatósági Osztály (case C-74/08) [2009] ECR I-3459 5.130

Pauls Agriculture Ltd v Council and Commission of the European Communities (case 256/81) [1983] ECR 1707 $3.074,3.092$

P. Dumortier frères SA and others v Council of the European Communities (joined cases 64 and 113/76, 167 and 239/78, 27, 28 and 45/79) [1982] ECR 1733 $1.025,1.032$, 3.044, 3.045, 3.046, 3.047, 3.059, 3.074, 3.084, 3.090, 3.099, 3.103, 3.111, 6.072, 9.014, 9.021, 9.023, 9.059

Peter Paul, Cornelia Sonnen-Lütte and Christel Mörkens v Bundesrepublik Deutschland (case C-222/02) [2004] ECR I-9425 6.018

Peterbroeck, Van Campenhout and Cie SCS v Belgian State (case C-312/93) [1995] ECR I-4599 $2.069,2.070,2.071,2.073,5.022,7.060,8.026$

Philip Morris Brands SARL and Others v Secretary of State for Health (case C-547/14) EU:C:2016:325 $2.041,2.043,2.046,2.050$

Pigs and Bacon Commission v McCarren and Co Ltd (case 177/78) [1979] ECR $2161 \ldots 2.021,2.029,4.007,5.008-5.010,5.012,5.064,5.098,5.126,5.167,5.171,5.172$

Pillbox 38 (UK) Ltd, trading as Totally Wicked v Secretary of State for Health (case C-477/14) EU:C:2016:324 $2.041,2.044$

Preston, Shirley and Others v Wolverhampton Healthcare NHS Trust and Others and Dorothy Fletcher and Others v Midland Bank plc (case C-78/98) [2000] ECR I-3201 2.066

Proceedings brought by Robert Pfleger and Others (case C-390/12) EU:C:2014:281 ... 2.046

Productores de Música de España (Promusicae) v Telefónica de España SAU (case C-275/06) [2008] ECR I-271 2.046 
Q-Beef NV v Belgische Staat and Frans Bosschaert v Belgische Staat, Vleesgroothandel Georges Goossens en Zonen NV and Slachthuizen Goossens NV (joined cases C-89/10 and C-96/10) [2011] ECR I-7819

Queen, The v Ministry of Agriculture, Fisheries and Food, ex parte: Hedley Lomas (Ireland) Ltd (case C-5/94) [1996] ECR I-2553

Queen, The v Secretary of State for the Environment and Ministry of Agriculture, Fisheries and Food, ex parte HA Standley and Others and DGD Metson and Others (case C-293/97) [1999] ECR I-2603 2.043

Queen, The v Secretary of State for Transport, ex parte: Factortame Ltd and others (case C-213/89) [1990] ECR I-2433 $2.004,2.019,2.027,2.029,2.060,5.016,6.009$ $6.026,7.023,8.016$

Queen, The (on the application of Delena Wells) v Secretary of State for Transport, Local Government and the Regions (case C-201/02) [2004] ECR I-723 $2.055,8.038$

Recheio - Cash \& Carry SA v Fazenda Pública/Registo Nacional de Pessoas Colectivas, and Ministério Público (case C-30/02) [2004] ECR I-6051 5.024

Reemtsma Cigarettenfabriken GmbH v Ministero delle Finanze (case C-35/05) [2007] ECR I-2425 $2.059,5.032,5.033,5.037,5.046,9.022$

Rewe-Handelsgesellschaft Nord $\mathrm{mbH}$ and Rewe-Markt Steffen v Hauptzollamt Kiel (Butter Buying Cruises) (case 158/80) [1981] ECR 1805 ........ 2.017, 2.018, 2021, 2.028, 2.053, 2.054, 2.056, 2.061, 2.068, 5.008, 5.014, 5.078, 5.081, 6.004, 7.013, 8.013, 8.041

Roman Angonese v Cassa di Risparmio di Bolzano SpA (case C-281/98) [2000] ECR I-4139 8.011

Roquette Frères SA v Direction des services fiscaux du Pas-de-Calais (case C-88/99) [2000] ECR I-10465 5.020

Rosalba Alassini v Telecom Italia SpA Filomena Califano v Wind SpA, Lucia Anna Giorgia Iacono v Telecom Italia SpA and Multiservice Srl v Telecom Italia SpA (Joined Cases C-317/08-C-320/08) [2010] ECR I-2213 ........ 2.006, 2.073, 5.027, 6.038, 7.060, 8.026

Rosalba Palmisani v Istituto nazionale della previdenza sociale (INPS) (case C-261/95) [1997] ECR I-4025 6.036, 6.037, 6.038, 6.039, 6.040

Ruckdeschel \& Co v Hauptzollamt Hamburg - St Annen and Diamalt AG v Hauptzollamt Itzehoe (joined cases 117/76 and 16/77) [1977] ECR 1753 3.085

SA Les Fils de Jules Bianco and J Girard Fils SA v Directeur Général des Douanes et Droits Indirects (joined cases 331/85, 376/85 and 378/85) [1988] ECR 1099 ...... 4.033, 5.012, 5.101-5.105, 5.106, 5.138, 5.141, 5.143, 5.151, 7.217, 9.045

SA Moulins \& Huileries de Pont-à-Mousson and Société coopérative Providence agricole de la Champagne v Office national interprofessionnel des céréales (joined cases 124/76 and 20/77) [1977] ECR 1795 $3.044,3.085,3.093$

Scarlet Extended SA v Société belge des auteurs, compositeurs et éditeurs SCRL (SABAM) (case C-70/10) [2011] ECR I-11959 $2.045,2.046$

Schwarz, Michael v Stadt Bochum (case C-291/12 0) EU:C:2013:670 ........................... 2.045

Seda Kücükdeveci v Swedex GmbH \& Co KG (case C-555/07) [2010] ECR I-365 .... 7.119, 8.038

Société Anonyme des Laminoirs, Hauts Fourneaux, Forges, Fonderies et Usines de la Providence and others v High Authority of the ECSC (joined cases 29, 31, 36, 39-47, 50, 51/63) [1965] ECR 911 $3.072,3.073,3.074$

Société Comateb and others v Directeur Général des Douanes et Droits Indirects (joined cases C-192/95-218/95) [1997] ECR I-165 .... 1.032, 4.033, 5.007, 5.014, 5.023, 5.037, 
5.041, 5.042, 5.072, 5.083, 5.106-5.116, 5.137, 5.138, 5.140, 5.142, 5.149, 5.152, 5.155,

$5.157,5.162,5.178,5.180,5.184,6.001,6.026,6.045,6.094,7.225,8.051,9.019,9.023$, $9.024,9.025,9.033,9.042,9.045$

Société d'Initiatives et de Coopération Agricole et al v Commission of the European

Communities (case 114/83) [1984] ECR 2598 $1.012,3.025$

Société Roquette frères v Commission of the European Communities (case 26/74) [1976]

ECR 677 $3.073,5.070$

SpA International Chemical Corporation v Amministrazione delle finanze dello Stato (case 66/80) [1981] ECR 1191 5.088-5.092

Streekgewest Westelijk Noord-Brabant v Staatssecretaris van Financiën (case C-174/02) [2005] ECR I-85 2.035

Sviluppo Italia Basilicata v Commission (case C-414/08 P) [2010] ECR I-2559 3.022

T-Mobile Netherlands BV, KPN Mobile NV, Orange Nederland NV and Vodafone Libertel NV v Raad van bestuur van de Nederlandse Mededingingsautoriteit (case C-8/08) [2009] ECR I-4529 7.129

TEAM Srl v Commission of the European Communities (case C-13/99 P) [2000] ECR I-4671 3.048

Test Claimants in the Thin Cap Group Litigation v Commissioners of Inland Revenue (case C-524/04) [2007] ECR I-2107 $5.007,6.054$

Test Claimants in the FII Group Litigation v Commissioners of Inland Revenue (case C-446/04) [2006] ECR I-11753 5.007, 5.045. 5.052, 5.054, 5.069, 6.054, 6.072, $8.042,8.047$

Texaco A/S v Middelfart Havn et al (joined cases C-114/95 and C-115/95) [1997] ECR I-4263 $5.014,5.024$

Thierry Delvigne v Commune de Lesparre-Médoc and Préfet de la Gironde (case C-650/13) EU:C:2015:648 2.042, 2.043, 2.044

Transportes Urbanos y Servicios Generales SAL v Administración del Estado (case C-118/08) [2010] ECR I-635 $2.065,2.066,2.067,5.019$

Trubowest Handel GmbH and Viktor Makarov v Council of the European Union and European Commission (case C-419/08 P) [2010] ECR I-2259 ....... 3.049, 3.050, 3.052, $3.060,3.068,4.007$

Unibet (London) Ltd and Unibet (International) Ltd v Justitiekanslern (case C-432/05) [2007] ECR I-2271 2.006, 2017, 2.020, 2.033, 5.047, 6.049, 7.060

Union royale belge des sociétés de football association ASBL v Jean-Marc Bosman, Royal club liégeois SA v Jean-Marc Bosman and others and Union des associations européennes de football (UEFA) v Jean-Marc Bosman (case C-415/93) [1995] ECR I-4921

Union syndicale-Service public européen and others v Council of the European Communities [1975] (case 72/74) [1975] ECR 401 $1.012,3.025$

VAG France SA v Établissements Magne SA (case 10/86) [1986] ECR 4071 .... 8.036, 8.038 van Duyn, Yvonne v Home Office (case 41/74) [1974] ECR 1337 $2.53,7.119$

Verholen, A and others v Sociale Verzekeringsbank Amsterdam (joined cases C-87/90, C-88/90 and C-89/90) [1991] ECR I-3757 $2.003,2.035,2.036,5.028$ Virginie Pontin v T-Comalux SA (case C-63/08) [2009] ECR I-10467 2.006 Volker und Markus Schecke GbR v Land Hesen (case C-92/09) [2010] ECR I-11063 $2.041,2.045$ 
von Colson, Sabine and Elisabeth Kamann v Land Nordrhein-Westfalen (case 14/83) [1984] ECR 1891 $2.008,2.023,2.024,2.034$

Walter Rechberger, Renate Greindl, Hermann Hofmeister and Others v Republik Österreich (case C-140/97) [1999] ECR I-3499 6.059-6.063, 6.071

WebMindLicenses Kft. v Nemzeti Adó-és Vámhivatal Kiemelt Adó és Vám Főigazgatóság (case C-419/14) EU:C:2015:832 $2.040,2.042$

Weber's Wine World Handels-GmbH and others v Abgabenberufungskommission Wien (case C-147/01) [2003] ECR I-11365 ... 2.062, 5.012, 5.117-5.122, 5.137, 5.140, 5.142, $5.143,5.152,5.157,5.184,5.185,6.091,7.060,9.023$

Werner Mangold v Rüdger Helm (case C-144/04) [2005] ECR I-9981 7.119

X (case C-318/13) EU:C:2014:2133 6.022

Zuckerfabrik Bedburg AG and others v Council and Commission of the European Communities (case 281/84) [1987] ECR 49 3.008

Zuckerfabrik Jülich AG v Hauptzollamt Aachen, British Sugar plc v Rural Payments Agency, an Executive Agency of the Department for Environment, Food and Rural Affairs and Tereos - Union de coopératives agricoles à capital variable v Directeur Général des douanes et droits indirects and Receveur principal des douanes et droits indirects de Gennevilliers (joined cases C-113/10, C-147/10 and C-234/10)

EU:C:2012:591 5.068

\section{General Court}

Aloys Schröder, Jan and Karl-Julius Thamann v Commission of the European Communities (case T-390/94) [1997] ECR II-501 3.011

Artegodan GmbH v European Commission (case T-429/05) [2010] ECR II-491 3.057

Association médicale européenne (EMA) v European Commission (case T-116/11)

EU:T:2013:634 4.023

Athanasios Pitsiorlas v Council of the European Union and European Central Bank (joined cases T-3/00 and T-337/04) [2007] ECR II-4779 3.052

Atlantic Container Line AB and Others v Commission of the European Communities (case T-113/04) EU:T:2007:377 3.060

Automec Srl v Commission of the European Communities (case T-64/89) [1990] ECR II-367 3.026

Blackspur DIY Ltd, Steven Kellar, J M A Glancy and Ronald Cohen v Council of the European Union and Commission of the European Communities (case T-168/94) [1995] ECR II-2627 $3.043,3.048,3.052$

Camar Srl and Tico Srl v Commission of the European Communities and Council of the European Union (joined cases T-79/96,T-260/97 and T-117/98) [2000] ECR II-2193 $3.076,3.081$

Cantina sociale di Dolianova Soc coop rl and Others v Commission of the European Communities (case T-166/98) [2004] ECR II-3991 $4.016,4.018$

CEVA Santé Animale SA and Pharmacia Entreprises SA v Commission of the European Communities (joined cases T-344/00 and T-345/00) [2003] ECR II-229 3.059

Confederazione Nazionale Coltivatori Diretti (Coldiretti) and 110 farmers v Council of the European Union and Commission of the European Communities (case T-149/96) [1998] ECR II-3841 $3.025,3.052$

Corus UK Ltd v Commission of the European Communities (case T-171/99) [2001] ECR II-2967 4.004, 4.006, 4.008, 4.009, 4.010, 4.011, 4.016, 5.067, 5.069 
DLD Trading Co v Council of the European Union (case T-146/01) [2003]

ECR II-6005

Dorsch Consult v Council and Commission (case T-184/95) [1998] ECR II-667 ......... 3.022

Eduardo Vieira, SA, Vieira Argentina, SA and Pescanova, SA v Commission of the

European Communities (joined cases T-44/01, T-119/01 and T-126/01) [2003] ECR

II-1209

Elena Candiotte v Council of the European Union (case T-108/94) [1996]

ECR II-87

Elliniki Viomichania Oplon AE (EVO) v Council of the European Union and Commission of the European Communities (case T-220/96) [2002] ECR II-2265

European Dynamics Luxembourg SA and others v OHIM (case T-299/11) EU:T:2015:757

Evropaïki Dynamiki - Proigmena Systimata Tilepikoinonion Pliroforikis kai Tilematikis AE v European Commission (case T-39/08) [2011] ECR II-437 3.080

FIAMM and FIAMM Technologies v Council of the European Union and Commission of the European Communities (case T-69/00) [2005] ECR II-5393

Frank Lamberts v European Ombudsman (case T-209/00) [2002] ECR II-2203 3.057

Fresh Marine Company A/S v Commission of the European Communities (case T-178/98) [2000] ECR II-3331

Guérin Automobiles v Commission of the European Communities (case T-195/95) [1997] ECR II-679 3.026. 3.027, 3.040, 3.114, 4.026, 7.013, 7.014

Günther Bühring v Council of the European Union and Commission of the European Communities (case T-246/93) [1998] ECR II-171

Hans-Martin Tillack v Commission of the European Communities (case T-193/04) [2006] ECR II-3995

Holcim (France) SA v Commission of the European Communities (case T-86/03) [2005] ECR II-1539

Idromacchine Srl, Alessandro Capuzzo and Roberto Capuzzo v European Commission (case T-88/09) [2011] ECR II-7833 $3.076,3.083$

İşçi Partisi (Turkish Labour Party) v Commission of the European Communities (case T-219/08) EU:T:2008:538 $2.003,3.025,3.036$

İşçi Partisi (Turkish Labour Party) v Commission of the European Communities (case T-223/09) EU:T:2009:524 $2.003,3.025,3.036$

Israel Institute of Technology and Technion Research \& Development Foundation Ltd v European Commission (case T-216/12) EU:T:2015:746 4.023

Jose Maria Sison v Council of the European Union (case T-341/07) [2011] ECR II-7915 3.057

Masdar (UK) Ltd v Commission of the European Communities (case T-333/03) [2006] ECR II-4377 $4.018,4.022$

Medici Grimm KG v Council of the European Union (case T-7/99) [2000] ECR II-2671 4.016

Mohamed Trabelsi and Others v Council of the European Union (case T-187/11) EU:T:2013:273

New Europe Consulting and Michael P Brown v Commission of the European Communities (case T-231/97) [1999] ECR II-2403 3.080

Odigitria AAE v Council of the European Union and Commission of the European Communities (case T-572/93) [1995] ECR II-2025 3.052 
Safa Nicu Sepahan Co v Council of the European Union (case T-384/11)

EU:T:2014:986 3.076

Schneider Electric SA v Commission of the European Communities (Schneider II) (case T-351/03) [2007] ECR II-2237 $3.063,3.064,3.065,3.066,9.011$

Stephen Sanders and Others v Commission of the European Communities (case T-45/01) [2004] ECR II-3315 $3.074,3.078$

Stephen Sanders and Others v Commission of the European Communities (case T-45/01) [2007] ECR II-2665 $3.074,3.078$

Stephen Sanders and Others v Commission of the European Communities (case T-45/01) [2009] ECR II-4093 $3.074,3.078$

T Port GmbH \& Co KG v Commission of the European Communities (case T-1/99) [2001] ECR II-415 3.052

Trubowest Handel GmbH and Viktor Makarov v Council of the European Union and Commission of the European Communities (case T-429/04) EU:T:2008:263 3.059

Vereniging van Exporteurs in Levende Varkens and others and Nederlandse Bond van Waaghouders van Levend Vee and others v Commission of the European Communities (joined cases T-481/93 and T-484/93) [1995] ECR II-2941 3.012

Yves Franchet and Daniel Byk v Commission of the European Communities (case T-48/05) [2008] ECR II-1585

\section{Civil Service Tribunal}

Allan Maxwell v European Commission (case F-55/09) EU:F:2010:44 4.023

\section{European Court of Human Rights}

Al-Sadoon and Mufdhi v the United Kingdom App no 61498/08 (ECtHR 2 March 2010) 2.026

Baczkowski and others v Poland App no1543/06 (ECtHR 3 May 2007) ........................ 2.026

Camenzind v Switzerland App no 21353/93 (ECtHR 16 December 1997) ..................... 2.026

Golder v the United Kingdom App no 4451/70 (ECtHR, 21 February 1975) ................ 2.033

Iatridis v Greece App no 31107/96 (ECtHR 25 March 1999) ......................................... 2.026

Iovchev v Bulgaria App no 41211/98 (ECtHR 2 February 2006) .................................... 2.027

Kamasinski v Austria App no 9783/82 (ECtHR 19 December 1989) .............................. 2.033

Messina v Italy (No 2) App no 25498/94 (ECtHR 28 September 2000) ......................... 2.026

The National \& Provincial Building Society, the Leeds Permanent Building Society and the Yorkshire Building Society v the United Kingdom Apps nos 21319/93, 21449/93, and 21675/93 (ECtHR, 23 October 1997) 2.050

Perez de Rada Cavanilles v Spain App no 28090/95 (ECtHR, 28 October 1998) ......... 2.043, $5.026,6.040$

Philis v Greece Apps nos 12750/87, 13780/88, and 14003/88 (ECtHR, 27 August 1991) 2.043

Shamayev and others v Georgia and Russia App no 36378/02 (ECtHR 12 April 2005) $2.026,2.027$

Silver and others v the United Kingdom Apps nos 5947/72; 6205/73; 7052/75; 7061/75; 7107/75; 7113/75; 7136/75 (ECtHR 25 March 1983) 
Stran Greek Refineries and Stratis Andreadis v Greece, App no 13427/87 (ECtHR, 9

December 1994) 2.050

Stubbings and others v the United Kingdom Apps nos 22083/93 and 22095/93 (ECtHR, 22 October 1996) $2.033,2.043$

Sunday Times v United Kingdom App no 6538/74 (ECtHR, 26 April 1976) 2.040

Swedish Engine Drivers' Union v Sweden App no 5614/72 (ECtHR 6 February 1976) 2.026

Winterwerp v the Netherlands App no 6301/73 (ECtHR, 24 October 1979) 2.043

\section{NATIONAL COURTS}

\section{Canada}

Air Canada v British Columbia (1989) 59 DLR (4th) 161 8.055

Kingstreet Investments Ltd v New Brunswick (Department of Finance) [2007] SCC 1 8.055

\section{Denmark}

U.1952.974:H (Supreme Court judgment) 9.059

\section{Sweden}

AD 2009 nr 89 (Swedish Labour Court) $8.003,8.019$

AD 2015 nr 70 (Swedish Labour Court) 8.037

NJA 2015 s 1072 (Supreme Court) 5.043

RÅ 2002 ref 108 (Administrative Supreme Court) 5.146

\section{USA}

Adams v Mills 286 US 397 (1932) $5.097,7.223,7.224$

Brunswick Corp v Pueblo Bowl-o-Mat Inc et al 429 US 477 (1977) 7.049

California et al v ARC America Corp et al 490 US 93 (1989) 7.102

Chattanooga Foundry \& Pipe Works v. City of Atlanta 203 US 390 (1906) 396 7.223

Clark Oil Co v Phillips Petroleum Co 148 F2d 580 (8th Cir 1945) 7.071

Hanover Shoe Inc v United Shoe Machinery Corp 392 US 481 (1968) ... 1.027, 3.102, 5.161, 5.180, 7.072, 7.073-7.076, 7.077-7.104, 7.111, 7.113, 7.214, 7.215, 7.216, 7.221, 7.222, $7.225,7.226,7.227,7.232,7.233,7.244,7.247,9.042$

Illinois Brick Co et al v Illinois et al 431 US, 7.116720 (1977) .... 3.032, 7.070-7.072, 7.074, $7.077-7.104,7.111,7.114,7.115,7.116,7.227,7.234,9.037$

Kansas \& Missouri v UtiliCorp United 497 US 199 (1990) 7.226

NorthWestern Oil v Socony-Vacuum Oil Co 138 F2d 967 (7th Cir 1943) 7.071

Southern Pac Co v Darnell-Taenzer Lumber Co 245 US 531 (1918) 3.102, 5.097, 5.180, 
Twin Ports Oil Co v Pure Oil Co 119 F2d 747 (8th Cir 1941) cert denied 314 US 644

(1941) $7.071,7.226$

\section{OPINIONS OF ADVOCATES GENERAL}

Amministrazione delle Finanze dello Stato v Denkavit Italiana Srl (case 61/79) [1980] ECR 1205, Opinion of AG Reischl $5.085,5.155$

Amministrazione delle Finanze dello Stato v SpA San Giorgio (case 199/82) [1983] ECR 3595, Opinion of AG Mancini ..... 3.107, 4.029, 5.083, 5.095-5.097, 5.098, 5.108, $5.149,5.150,5.155,5.157,5.158,5.159,5.161,7.126,8.046,9.045$

Birra Wührer SpA and others v Council and Commission of the European Communities (joined cases 256, 257, 265, 267/80, 5 and 51/81 and 282/82) [1984] ECR 3693, Opinion of AG verLoren van Themaat 3.094, 3.101, 3.109, 3.110

Brasserie du Pêcheur SA v Bundesrepublik Deutschland and The Queen v Secretary of State for Transport, ex parte: Factortame Ltd and others (joined cases C-46/93 and C-48/93) [1996] ECR I-1029, Opinion of AG Tesauro $6.018,6.029,6.070$

Combinatie Spijker Infrabouw-De Jonge Konstruktie and Others v Provincie Drenthe (case

C-568/08) [2010] ECR I-12655, Opinion of AG Cruz Villalón 6.087

Commission of the European Communities v Italian Republic (case C-129/00) [2003] ECR

I-14637, Opinion of AG Geelhoed 5.142

Compagnie Continentale France v Council of the European Communities (case 169/73)

[1975] ECR 117, Opinion of AG Trabucchi 3.053

Courage Ltd v Bernard Crehan and Bernard Crehan v Courage Ltd and Others (case C-453/99) [2001] ECR I-6297, Opinion of AG Mischo $7.015,7.170-7.172$

Danfoss A/S and Sauer-Danfoss ApS v Skatteministeriet (case C-94/10) [2011] ECR I-9963, Opinion of AG Kokott ... 3.068, 5.007, 5.029, 5.031-5.036, 5.047, 5.048, 5.054, $6.044,6.072,6.076,6.077,6.078,8.046,9.010,9.011$

Danske Slagterier v Bundesrepublik Deutschland (case C-445/06) [2009] ECR I-2119, Opinion of AG Trstenjak $6.034,6.070$

DGV, Deutsche Getreideverwertung und Rheinische Kraftfutterwerke GmbH and others v Council and Commission of the European Communities (joined cases 241, 242, 245 to 250/78) [1979] ECR 3017, Opinion of AG Capotorti 9.021

Fantask A/S ea v Industriministeriet (Erhvervministeriet) (case C-188/95) [1997] ECR

I-6783, Opinion of AG Jacobs 6.029

Francovich and Bonifaci and others v Italian Republic (joined cases C-6/90 and 9/90) [1991]

ECR I-5357, Opinion of AG van Gerven 6.010

Grand Duchy of Luxemburg v Berthe Linster, Aloyse Linster and Yvonne Linster (case

C-287/98) [2000] ECR I-6917, Opinion of AG Léger 8.038

Hans Just I/S v Danish Ministry for Fiscal Affairs (case 68/79) [1980] ECR 501, Opinion of AG Reischl $5.078-5.081,5.155$

H J Banks \& Co Ltd v British Coal Corporation (case C-128/92) [1994] ECR I-1209, Opinion of AG van Gerven $7.013,7.052,8.017$

Interquell Stärke-Chemie GmbH and Diamalt AG v Council and Commission of the European Communities (joined cases 261 and 262/78) [1979] ECR 3045, Opinion of AG Capotorti 9.021

xxviii 
Ireks-Arkady GmbH v Council and Commission of the European Communities (case 238/78) [1979] ECR 2955, Opinion of AG Capotorti 3.071, 3.072, 3.073, 3.079, 3.085-3.090, 3.092, 3.102, 3.109, 7.1126, 9.021, 9.030

Kone AG and Others v ÖBB-Infrastruktur AG (case C-557/12) EU:C:2014:1317, Opinion of AG Kokott $7.036,7.050,7.137-7.140$

Lady \& Kid A/S and Others v Skatteministeriet (case C-398/09) [2011] ECR I-7375, Opinion of AG Cruz Villalón $5.123,5.125,5.126,5.172,5.174$

Marshall, M Helen v Southampton and South-West Hampshire Area Health Authority (Marshall II) (case C-271/91) [1993] ECR I-4367, Opinion of AG van Gerven

Masdar (UK) Ltd v Commission of the European Communities (case C-47/07 P) [2008] ECR I-9761, Opinion of AG Mazák

Ministre du Budget, des Comptes publics et de la Fonction publique v Accor SA (case C-310/09) [2011] ECR I-8115, Opinion of AG Mengozzi 5.007

Pauls Agriculture Ltd v Council and Commission of the European Communities (case 256/81) [1983] ECR 1707, Opinion of AG Mancini

P. Dumortier frères SA and others v Council of the European Communities (joined cases 64 and 113/76, 167 and 239/78, 27, 28 and 45/79) [1982] ECR 1733, Opinion of AG Capotorti 9.021

Rewe-Zentralfinanz eG and Rewe-Zentral AG v Landwirtschaftskammer für für das Saarland (case 33/76) [1976] ECR 1989, Opinion of AG Warner 8.041

SA Les Fils de Jules Bianco and J Girard Fils SA v Directeur Général des Douanes et Droits Indirects (joined cases 331/85, 376/85 and 378/85) [1988] ECR 1099, Opinion of AG Sir Gordon Slynn 9.045

Rosalba Alassini v Telecom Italia SpA Filomena Califano v Wind SpA, Lucia Anna Giorgia Iacono v Telecom Italia SpA and Multiservice Srl v Telecom Italia SpA (joined cases C-317/08-C-320/08) [2010] ECR I-2213, Opinion of AG Kokott 2.006

Société Comateb and others v Directeur Général des Douanes et Droits Indirects (joined cases C-192/95-218/95) [1997] ECR I-165, Opinion of AG Tesauro ....... 5.023, 5.083, 5.108. 5.109-5.111, 5.149, 5.150, 5.155, 5.157, 5.158, 5.178, 7.236, 9.042, 9.045

VAG France SA v Établissements Magne SA (case 10/86) [1986] ECR 4071, Opinion of AG Mischo 8.036

Vincenzo Manfredi v Lloyd Adriatico Assicurazioni SpA, Antonio Cannito v Fondiaria Sai $\mathrm{SpA}$ and Nicolò Tricarico and Pasqualina Murgolo v Assitalia SpA (joined cases C-295/04-C-298/04) [2006] ECR I-6619, Opinion of AG Geelhoed $5.142,7.178$ 